\title{
First-year Experience in New Postgraduate Clinical Training System
}

\author{
Shigeaki Aoyagi, MD. \\ Advisory Editorial Board, Japanese Association for Thoracic Surgery, Kurume University School of Medicine
}

The Japanese Ministry of Health, Labor, and Welfare has introduced a new 2-year postgraduate clinical training system for medical residents to learn basic diagnostic and therapeutic knowledge and techniques and basic life support procedures for patients, or "primary care," in April 2004. This system requires that all residents receive at least 6 months of training in internal medicine and 3 months of training in surgery, emergency care medicine or anesthesiology, pediatrics, obstetrics and gynecology, psychiatry and mental health, and public health during the first 2 years after graduating from a medical department.

Our hospital instituted a 2 -year rotating program that includes 6 months of training in internal medicine and 3 months each in surgery and emergency care medicine or anesthesiology during the first year of study. In the year that has passed since the program was introduced, problems have arisen. The goal of the 3-month training in surgery for residents is, we believe, to learn basic pathogenesis and pathophysiology of diseases, preoperative and postoperative management for patients, including biologic reactions to surgical stress, and basic surgical techniques common to all fields of surgery rather than specialized surgical training. Residents at our department of surgery take 3 months of training in one of 5 surgical subspecialties of their choice-cardiovascular, respiratory, gastrointestinal, hepato-biliary-pacreatic, and breast and endocrine surgery. Three months is not enough, however, for them to learn all subjects required for surgical training. It is not always possible, either, for them to cover all subjects that only one surgical subspecialty requires during the training period. Based on our one year of experience, then, it appears difficult for residents to meet all requirements in surgical training. This raises the question of just how useful and meaning-

From Department of Surgery, Kurume University School of Medicine, Fukuoka, Japan.

Address for reprints: Shigeaki Aoyagi, MD, Department of Surgery, Kurume University School of Medicine, 67 Asahi-machi, Kurume, Fukuoka 830-0011, Japan. ful this training system is for residents. The second year of training includes 3 months in psychiatry and mental health and public health. Given the goal of this system, I believe that such subjects are less important than training in internal medicine and surgery and that studying such fields requires a certain amount of knowledge of the pathogenesis and pathophysiology of all sorts of diseases and technical experience as a general physician. In my opinion, it would be more meaningful and substantive for residents to spend more time on training in internal medicine or surgery during the first 2 years rather than attempting to train in psychiatry and at public health centers. I therefore feel that it is necessary to upgrade and reconsider training periods and methods.

A great problem also lies in the case of attending physicians chosen to serve as training instructors. Attending physicians should be aware that clinical training is held for the sake of patients who will be treated by residents who finish the training course, not for the sake of the residents themselves or for their hospitals. In actual practice, most attending physicians are responsible for large numbers of patients and are, for the sake of their futures, required to acquire more knowledge and surgical techniques. They must also engage in new research. Again, however, attending physicians having to teach residents under the new system have no time to pursue their own interests, and this important role has not yet been evaluated sufficiently. It is therefore necessary, I think, to clarify and evaluate the needs of attending physicians.

From the view of thoracic and cardiovascular surgery, this new training system has introduced a serious labor shortage. Greater burdens have been placed on attending physicians and reduced the time they need to instruct residents appropriately. This situation will, I fear, make residents less eager to major in surgery, particularly thoracic and cardiovascular surgery, and thereby reduce the number of would-be surgeons. In fact, according to a survey we conducted among residents after this first year of training, the consensus was that the number of residents who hoped to major in surgery has dropped 
compared to before the training system was introduced. I therefore believe that we must propose a more substantive and practical training program for residents who finish the initial training program to keep them from losing their interest in thoracic and cardiovascular surgery. 